\title{
Integrated Thermal Insulation Packing and Vehicle Routing for Perishable Products in Community Group Purchase
}

\author{
Wenbing Shui (iD, Huimin Zhao $(\mathbb{D}$, and Mengxia Li $(\mathbb{D}$ \\ Kunming University of Science and Technology, Kunming 650500, China \\ Correspondence should be addressed to Huimin Zhao; 20192206017@stu.kust.edu.cn
}

Received 19 November 2020; Revised 18 March 2021; Accepted 26 March 2021; Published 15 April 2021

Academic Editor: Tingsong Wang

Copyright (C 2021 Wenbing Shui et al. This is an open access article distributed under the Creative Commons Attribution License, which permits unrestricted use, distribution, and reproduction in any medium, provided the original work is properly cited.

\begin{abstract}
Two transportation modes have been used to solve the "last kilometer" delivery problem of perishable products in China. One mode involves the use of refrigerated vehicles, and the other involves transportation with nonrefrigerated vehicles but with thermal insulation packaging and phase-change refrigerants. In this paper, we studied the distribution of fresh products using nonrefrigerated vehicles under the community group purchase model. A new integrated model that can simultaneously select insulation packaging methods and vehicle paths was developed. We designed a heuristic algorithm based on genetic algorithm to solve larger-scale problems. We found that nonrefrigerated vehicle delivery was better than refrigerated vehicle delivery when neither the cost of the phase-change refrigerant nor the cost of packaging accounts for more than $50 \%$ of the total cost. There was an optimal balance between insulation packaging cost and shipping cost. The combination of thin and light packaging materials and high-efficiency cold storage materials could achieve the goals of ensuring the quality of fresh products and reducing transportation costs simultaneously.
\end{abstract}

\section{Introduction}

As a new mode of social e-commerce, community group purchases are developing rapidly with the help of social software and other social traffic portals. Most users use community group purchase to buy perishable products such as vegetables and fruits. Temperature is the most important environmental factor affecting products' deterioration rate and postharvest life for these products, with higher temperatures providing an environment for bacterial growth and resulting in a shortened shelf life. To ensure the delivery quality and freshness of fresh agricultural products, ensuring complete cold chain transportation is key.

At present, two transportation modes are usually used to solve the "last kilometer" delivery problem. One mode involves the use of refrigerated vehicles to transport fresh agricultural products, and the other involves transportation with conventional nonrefrigerated vehicles but with thermal insulation packaging and phase-change refrigerants. There are high operating costs for the use of refrigerated vehicles. Besides, nonrefrigerated vehicles can cost less than refrigerated vehicles, and logistics companies have more choices in selecting different vehicle types. This allows greater flexibility in delivery, for example, the mixing of perishable products with other categories. Therefore, this transportation model is more appropriate than the refrigerated vehicle mode for filling the various and small product orders demanded by individual consumers.

Thermal insulation packaging plays an important role when we consider the nonrefrigerated vehicle transportation mode. The complex interaction between package selection and vehicle route optimization is reflected in the following two aspects. On the one hand, the phase transition temperature of the refrigerant determines the temperature boundary of product delivery in transit. The maximum allowable delivery temperature is codetermined by the initial quality, delivery time, and final quality requirement. Therefore, we focus on the quality of the phase-change refrigerant, which guarantees that the phase transition temperature is lower than the maximum allowable delivery temperature. On the other hand, the insulation packaging material determines the thermal resistance, which in turn 
determines the insulation duration. At the same time, the insulation duration is also affected by vehicle routing. In summary, coordination between packaging selection and vehicle routing optimization is important.

Most published research on collaborative optimization has focused on common research topics such as the locationrouting problem (LRP), production routing problem (PRP), production-distribution problem (PDP), and inventory routing problem (IRP). However, studies in this field on joint optimization of packaging systems and vehicle route planning are lacking because many package-dependent costs are frequently overlooked in logistics activities.

Compared with the existing works, our main contributions of this study are summarized as follows. This work studied the distribution of fresh agricultural products from a new perspective. It integrated the multitype thermal insulation packaging selection problem of nonrefrigerated vehicles into the vehicle routing problem (VRP). Another main contribution was that this paper designed a heuristic algorithm that can quickly solve large-scale problems. On the basis of the above research, this paper analyzed the benefits of this nonrefrigerated vehicle mode but with thermal insulation packaging and phase-change refrigerants. We aimed to reduce the logistics cost while guaranteeing the delivery quality of fresh food. This paper provides a theoretical basis for the incorporation of multiple packaging selection into the VRP.

The remainder of this paper is organized as follows: Section 2 introduces the related research review. Section 3 introduces the descriptions and assumptions of the problem and establishes relevant models and design algorithms. Section 4 presents some examples to verify the model and algorithm. Meanwhile, we analyze the interaction effect among the main parameters. Finally, we summarize the major conclusions of our study and provide several suggestions for further research.

\section{Related Works}

In this section, we introduce previous research on community group purchases, thermal insulation packaging, and distribution of perishable products.

\subsection{Literature about Community Group Purchases.} Community group purchases are a new business model, and research on them is scarce. Proper pricing mechanisms are conducive to higher profits under community group purchase models. Li et al. [1] developed two-stage pricing mechanisms to evaluate the impact of competition and waiting costs on the profitability and efficiency of community-based group purchases.

The intention to participate in community group purchases has received some attention among researchers, particularly in recent years. Li et al. [2] constructed a theoretical model and questionnaire on community group purchase consumer participation behavior based on the theory of stimulus-organism-response (SOR) and commitment trust theory.
In addition, some researchers have addressed the distribution of perishable products in the context of CGPs. Liu et al. [3] proposed a balanced scorecard to construct an index system to evaluate and select suitable distribution modes based on the analysis of community group purchase distribution of fresh agricultural products. Wang and Qiu [4] improved the VRP model by incorporating the minimum total cost, including fixed costs, transportation costs, and penalty costs, under the constraint function of the distribution time window for community group purchases. They concluded that the suggested approach is computationally very efficient and provides a reference for decision-making in fresh community group purchase enterprises.

2.2. Literature about Thermal Insulation Packaging and Phase-Change Refrigerants. Thermal insulation packaging is critical to maintaining low temperatures for the perishable products. Lee and Yoo [5] discovered that the higher the thermal insulation capacity of packaging was, the longer the changes in the physical properties of frozen cooked rice were delayed. Fang et al. [6] also indicated that the thermal insulation effect was influenced by packaging material. $\mathrm{Li}$ et al. [7] presented that different choices between more expensive and less expensive packaging lead to different sales, cost, and waste levels, and these different choices require different inventory control policies.

Simultaneously, the use of refrigerants during transportation by nonrefrigerated vehicles is another important research topic. Oró et al. [8] found that frozen product temperatures remained at lower values much longer when phase-change materials were employed. Singh [9] presented that the temperature control for perishable products during shipping can be improved with the use of phase-change materials. These studies can provide a theoretical basis for the transport of perishable products with nonrefrigerated vehicles.

\subsection{Literature about Perishable Product Distribution.} Perishable products require complete cold chain transportation for the best retention of product value and appearance. At present, most research covers refrigerated modes of perishable food product delivery. Amorim and Almada-Lobo [10] proposed a model that decouples minimization of distribution costs from maximization of the freshness state of the delivered products. Hsu et al. [11] considered the randomness of the perishable food delivery process with time windows to obtain optimal delivery routes, loads, fleet dispatching, and departure times for the delivery of perishable food products. Rabbani et al. [12] established a VRP model that considered minimization of transportation costs and a minimum freshness level to improve profit in the context of different numbers of depots. They considered different scenarios and described the routing problem based on the traditional transportation method.

However, this area of study lacks research on the use of nonrefrigerated vehicles for transportation of fresh products. Many enterprises have started to use nonrefrigerated 
vehicles to transport fresh products because they allow greater flexibility in delivery. Byung and Young [13] presented a VRP that encompasses both refrigerated and general vehicles for multicommodity perishable food product delivery. This aim was to confirm the performance and availability of the refrigerated transport mode for perishable food product delivery in comparison with the general mode. However, the paper focused on specific vehicle routing, and packaging was not considered. Dieckmann et al. [14] designed new thermal insulation packaging materials made from feathers for nonrefrigerated vehicle transport. They concluded that feather materials have the potential to replace the materials currently used for delivery of perishable food products.

To the best of the authors' knowledge, Li et al. [15] offered the only study analyzing multiple types of packaging in the perishable food PRP. They pointed out that different packaging selections lead to different product shelf lives and influence the selling price. However, they used a pattern that is not applicable to community group purchases, while considering the complex relationships among quality of phase-change refrigerants, the types of packaging, and vehicle routing.

The current paper provides a new research perspective compared with the work of Shui and Li [16], which are the most recent literature relevant to the topic of our interest. The paper by Shui and Li [16] constructed a pricing model for community group purchase and a distribution costoptimal model based on refrigerated vehicles. Meanwhile, they proposed a collaborative optimization mechanism of the pricing model and distribution model based on bilevel programming theory. Refrigeration cost, fuel cost, and fixed cost were taken into consideration in the refrigerated vehicle distribution model, while in this paper, we propose a nonrefrigerated vehicle distribution model based on the VRP problem, considering the choice of insulation packaging mode. In this model, the costs include fixed cost, fuel, packaging material, and coolant cost. In terms of algorithms, we extend initial work by designing a heuristic algorithm based on a genetic algorithm to obtain the optimal solution and solve by MATLAB, while the model in the other paper is solved by the exact algorithm and tested by LINGO. In terms of conclusion, Shui and $\mathrm{Li}$ [16] found that collaborative optimization mechanism of pricing and distribution can improve the profit level of community group purchase, while we found the specific conditions when nonrefrigerated vehicle distribution cost is lower than refrigerated vehicles.

In this study, we focus on multitype packaging selection in the context of transport of perishable produce in nonrefrigerated vehicles. To address the freshness issue, we consider the maintenance of low temperatures with thermal insulation packaging and phase-change refrigerants. Because community group purchases can aggregate customer demand, we assume that the delivery service at each point uses only one type of integrated packaging and establish a profit maximization model. In other contexts, the use of such a model may lead to packaging cost larger than distribution cost.

\section{Materials and Methods}

3.1. Problem Description and Assumption. Reducing logistics costs and optimizing distribution networks are essential for community group purchase enterprises, and perishable product quality continuously changes after the production stage. Thermal insulation packaging and phase-change refrigerants are needed to maintain a certain temperature when conventional nonrefrigerated vehicles are used to deliver perishable food products. The choice of packaging type and refrigerant quality influence the delivery quality of perishable food products, which is related to customer satisfaction. Therefore, insulation package selection is directly related to the profit level of community group purchase enterprises. The problem that we focus on is that when community group purchase enterprises receive orders, the distribution center begins to choose the appropriate package for each demand point to meet the insulation requirement based on different arrival times for each conventional nonrefrigerated vehicle. Each vehicle services the demand points in turn and delivers the perishable products to the head of the community group.

The integration of multipackage selection into the VRP is defined on a complete digraph $G=\{N, A\}$. The set $N$ of nodes is comprised of the subset $N=\{i \mid i=0,1,2, \ldots, L\}$, which indicates that there are $L$ demand nodes and one distribution center " 0 ." Let $A=(i, j)$ denote the set of arcs that link all nodes from $i$ to $j$. In this paper, we establish the following assumptions:

(1) There is only one distribution center with different types of conventional nonrefrigerated vehicles.

(2) Because different types of fresh agricultural products have different distribution requirements, this paper considers only distribution of a single product.

(3) The location coordinates of the distribution center and the demand nodes are known.

(4) Each order quantity of each demand node is known, and each node can be visited once by one vehicle.

(5) There is no time limit on the service time of each demand point.

(6) Only one packaging type can be chosen for each demand point.

(7) The remaining perishable products must be sent to the head of the community group before phase change occurs in the refrigerant.

3.2. Objective Function and Constraints. We propose a mathematical model integrating package selection into the VRP, whereby a fresh community group purchase enterprise chooses the type of thermal insulation packaging as well as the optimal vehicle route to deliver the products to the demand points. We first derive formulations (6) and (9) before establishing the model.

Formula (6) derivation process is as follows.

Fourier [17] discovered that there is a linear relationship between the amount of thermal conductivity $\lambda$, the area of 
thermal conductivity $A$, the temperature difference between the two sides of the wall $\Delta T$, and the thickness of the wall $\delta$.

$$
Q=\lambda A \frac{\Delta T}{\delta} .
$$

In equation (1), $\lambda$ is the heat conductivity coefficient of the material, which reflects the strength of the material's thermal conductivity; the greater the coefficient of thermal conductivity is, the better the thermal conductivity of the material is. At the same time, the different thermal resistances $R$ correspond to different conductivities $\lambda$ for multiple types of packaging. The relationship between the two is shown as follows:

$$
R=\frac{\delta}{\lambda}
$$

The temperature of the latent heat accumulator changes little in the process of phase change, so the temperature in the incubator can be regarded as approximately constant. According to equations (1) and (2), we can obtain equation (3), which is the heat transfer from the thermal insulation packaging $b$ in transit:

$$
Q=\frac{3600 t_{i}\left(T_{s}-T_{b}^{0}\right)}{R}, \quad \forall i \in N, b \in B
$$

In equation (3), $T_{S}$ is the environmental temperature, $T_{b}^{0}$ is the temperature of insulation packaging $b$ before the complete phase change of the refrigerant accumulator, and $t_{i}$ is the time when the delivery service arrives at point $i$. It is calculated by equation (4):

$$
t_{j}=\sum_{k=1}^{V} \sum_{i=0}^{L}\left(t_{i}^{k}+\frac{d_{i j}}{v_{i j}}+\frac{q_{i}}{v_{0}}\right) y_{i j}^{k}, \quad j \geq 1
$$

In equation (4), $t_{i}^{k}$ is the time when vehicle $k$ arrives at customer node $i$, and $t_{0}^{k}$ denotes that vehicle $k$ departs from the distribution center. Here, $d_{i j}$ is the distance between customer node $i$ and customer node $j . v_{i j}$ is the average speed from customer node $i$ to customer node $j . v_{0}$ denotes the loading and unloading speed at customer node $i . q_{i}$ is the demand at node $i$.

$$
Q=1000 \mathrm{ml}
$$

Equation (5) denotes the amount of heat absorbed by a refrigerant during phase change, $m$ is the quality of the refrigerant, and $l$ is the phase-change latent heat of the refrigerant. We can derive equation (6) when equation (3) is equal to equation (5).

$$
Q_{i}^{b}=\frac{3600\left(T_{s}-T_{b}^{0}\right) t_{i} A_{i}^{b}}{1000 L_{b} R_{b}}, \quad t_{i}=t_{j}, \forall i \in N, b \in B .
$$

Equation (6) represents the quality of refrigerant $Q_{i}^{b}$ by the refrigerant accumulator during a phase change to maintain a low temperature during transit to customer node $i$ with packaging type $b, L_{b}$ is the phase-change latent heat of the refrigerant with packaging type $b$, and $R_{b}$ is the thermal resistances with packaging type $b$, while $A_{i}^{b}$ denotes the total package area of package $b$ at customer node $i$, and it is calculated by equation (7).

$$
A_{i}^{b}=q_{i} \beta_{b}, \quad \forall i \in N, b \in B .
$$

In equation (7), $\beta_{b}$ is the area of category $q_{i}$ packaging covered by product per unit volume.

Formula (9) derivation process is as follows.

Arrhenius [18] proposed the Arrhenius equation (8), which reflects the changes in the relationship between the chemical reaction rate constant and temperature.

$$
\begin{aligned}
K & =A \cdot e^{(-E a / r T)}, \\
M_{k} & =M_{0}-K t .
\end{aligned}
$$

In equation (8), $A$ refers to the prefactor or rate constant. Here, $E a$ denotes activation energy. $r$ represents the gas constant. $T$ denotes the thermodynamic temperature. Equation (9) represents that the initial quality $M_{0}$ varies linearly with time $t$. Combining formulas (8) and (9), we can deduce formula (10) to ensure the maximum temperature limits $T_{i}$ of fresh agricultural delivery quality.

$$
T_{i}=\frac{E_{a}}{R \ln \left(A t_{i} /\left(M_{0}-M_{k}\right)\right)}-273.15, \quad \forall i \in N, i \geq 1 \text {. }
$$

On the above basis, the model is as follows:

$$
\operatorname{Max} p \sum_{i=1}^{L} q_{i} z_{i}-\sum_{k=1}^{V} \sum_{i=0}^{L} \sum_{j=1}^{L} c_{i j}^{k} y_{i j}^{k}-\sum_{b=1}^{B N} s_{b}-\sum_{k=1}^{V} f_{k} O_{k} .
$$

Formula (11) is the objective function, which aims to maximize profit, considering the total cost including transportation costs, the fixed cost of dispatching vehicles, and thermal insulation packaging and phase-change refrigerant costs. $p$ is the given price of a single product. The binary variable $Z_{i}$ is equal to 1 when community node $i$ is delivered, and binary variable $y_{i j}^{k}$ is equal to 1 when vehicle $k$ visits node $j$ immediately after node $i$. Binary variable $O_{k}$ is equal to 1 when vehicle $k$ is used. $s_{b}$ represents the total packaging and refrigerant costs, which is calculated by equation (12). $c_{i j}^{k}$ denotes the fuel cost of transportation from $i$ to $j$ by vehicle $k$, and it is calculated by equation (13). $f_{k}$ denotes the fixed cost of vehicle $k$.

$$
\begin{aligned}
s_{b}= & c_{b}^{1}+c_{b}^{2}, \quad b \in B, \\
c_{i j}^{k}= & a_{0}^{k} \times\left(b_{0}^{k}+c_{0}^{k} . w_{i}^{k}\right) d_{i j} f, \\
& \forall i, j \in N, j \neq 0, k \in K .
\end{aligned}
$$

In equation (12), let $c_{b}^{1}$ denote the packaging cost of using package $b$, which is the relative packing area and is calculated by equation (14). $c_{b}^{2}$ denotes the refrigerant costs of using package $b$, which is relative to the quality of the refrigerant and is calculated by equation (15). In equation (13), $c_{i j}^{k}$ is relative to $w_{i}^{k}$, which is the weight of vehicle $k$ leaving customer $i$, and $w_{i}^{k}$ is shown in equation (16), where $a_{0}^{k}, b_{0}^{k}$, 
and $c_{0}^{k}$ are conversion parameters for vehicle $k$ and $f$ represents the unit fuel rate.

$$
c_{b}^{1}=\sum_{i=1}^{L} q_{i} \beta_{b} z_{i} U_{i}^{b} g_{b}, \quad \forall i \in N, b \in B .
$$

In equation (14), $\beta_{b}$ denotes the conversion parameter between the area of $b$ and unit product quality. The binary variable $U_{i}^{b}$ is equal to 1 if packaging type $b$ is used in customer node $i . g_{b}$ denotes the per unit area cost of packaging type $b$.

$$
c_{b}^{2}=\sum_{i=1}^{L} Q_{i}^{b} U_{i}^{b} h_{b}, \quad \forall i \in N, b \in B .
$$

In equation (15), $h_{b}$ denotes the unit cost of the refrigerant.

$$
w_{i}^{k}=w_{k}+\sum_{i=1}^{L} q_{i} x_{i}^{k}=w_{k}+\sum_{i=1}^{L} \sum_{j=1}^{L} q_{i} y_{i j}^{k}, \quad \forall k \in K .
$$

In equation (16), $w_{k}$ denotes the weight of vehicle $k$, and binary variable $x_{i}^{k}$ is equal to 1 when vehicle $k$ visits customer node $i$.

Constraints (17) to (21) are used to represent the routing flow. Constraint (17) ensures that there is only one delivery service for each demand node. Constraint (18) indicates that each route starts and ends at the same demand node. Constraints (19) to (20) are used to ensure that if a vehicle is used, it must depart from and end up at the depot. Constraint (21) is designed to eliminate the subloop. Constraint (22) ensures that no route has total community demand exceeding the capacity of vehicle $k$. Constraint (23) is used to represent the relationship between $x_{i j}$ and $y_{i}^{k}$. Constraint (24) aims to ensure that only one type of packaging can be selected for each demand node. Constraint (25) represents the relationship between $Q_{i}^{b}$ and $U_{i}^{b}$ that ensure that the value of quality of refrigerant is reasonable. Constraint (26) provides the maximum boundary of the product temperature when the delivery service arrives at demand node $i$. Constraint (27) aims to ensure that the temperature of the thermal insulation cannot exceed the temperature limits for delivery.

$$
\begin{aligned}
z_{i}-\sum_{k=1}^{V} x_{i}^{k} & =0, \quad \forall i \in N, i \geq 1, \\
\sum_{i=0}^{L} y_{i p}^{k}-\sum_{j=0}^{L} y_{p j}^{k} & =0, \\
\forall k \in K, p \in N, y_{i j}^{k} & =0, \forall i, j \in N, i=j, k \in K, \\
O_{k}-\sum_{p=1}^{L} y_{0 p}^{k} & =0, \quad \forall k \in K, \\
O_{k}-\sum_{p=1}^{L} y_{p 0}^{k} & =0, \quad \forall k \in K,
\end{aligned}
$$

$$
u_{i}-u_{j}+L y_{i j}^{k} \leq L-1, \quad u_{i}, u_{j} \geq 0, i, j \in N, i \neq j, j \geq 2
$$

$$
\sum_{i=1}^{L} \sum_{j=1}^{L} q_{i} y_{i j}^{k} \leq G_{k} O_{k}, \quad \forall k \in K
$$

$$
\sum_{i=1}^{L} q_{i} x_{i}^{k}=\sum_{i=0}^{L} \sum_{j=1}^{L} q_{j} y_{i j}^{k}, \quad \forall k \in K
$$

$$
\sum_{b=1}^{B N} U_{i}^{b}=Z_{i}, \quad \forall i \in N, i \geq 1
$$

$$
Q_{i}^{b} \leq \operatorname{Big} \cdot U_{i}^{b}, \quad \forall i \in N, i \geq 1, b \in B,
$$

$$
T_{i}=\frac{E_{a}}{R \ln \left(A t_{i} /\left(M_{0}-M_{k}\right)\right)}-273.15, \quad \forall i \in N, i \geq 1,
$$

$$
T_{i} \geq T_{b}^{0} Z_{i}, \quad i \in N, i \geq 1, b \in B .
$$

3.3. Algorithm Design. The VRP problem has been proven to be an NP-hard problem, with many constraints and a complex objective function. There are two common methods for solving this problem: heuristic methods and exact procedures. Exact procedures always have a limited range of applications in practice, so heuristic algorithms are most often used to solve routing problems. In the heuristic algorithm, the best-known result for VRPs has been obtained using Tabu search or simulated annealing. Genetic algorithms (GAs) have seen widespread application to various combinatorial optimization problems, such as certain types of VRPs. The heuristic algorithm base on GA is adopted in this paper because this paper considers the choice of packaging mode in traditional VRP.

The distribution schedule in our problem has to satisfy both the temperature constraints and load-capacity constraints for vehicles arriving at the demand nodes. However, it is difficult to construct a method of solving the above two constraints simultaneously. In this paper, we use a heuristic algorithm based on the genetic algorithm to obtain the solution to this problem. First, we use the natural numbers for encoding. For each of these chromosomes, we can calculate the corresponding delivery time and temperature requirement, and through decoding, we can obtain the vehicle selection. Furthermore, we choose different packaging types under the constraints of fresh product delivery time, initial quality, and requirements. Based on the packaging selected as above, we can calculate the quality of the refrigerant and the refrigerant and packaging costs. Finally, in these populations, we choose the individuals with the lowest cost-including fixed cost, packaging cost, and transportation cost - to carry out crossover, mutation, and selection until the optimal solution is found. The specific flowchart is shown in Figure 1. 
The detailed contents of the heuristic algorithm base on GA are as follows.

3.3.1. Chromosome Structure. The solution to our problem includes vehicle route planning, vehicle type selection, and packaging selection. Therefore, we use natural numbers to encode the decision variables in the model, which represents the service order of demand nodes. The vehicle starts from the depot and provides delivery service for demand nodes when the total weight of the perishable goods at these distribution points is within the capacity limit of the vehicle. If this weight exceeds the capacity limit of the vehicle, the vehicle returns to the depot, and the next vehicle starts to distribute from point $j$ until all demand points are serviced. To derive the highest profit in this process, we choose to forgo provision of delivery services when the distribution cost is higher than the cost of abandoning the delivery. This option is coded as " 0 ." To express the relationship between route and vehicle choice, we use the structure of the chromosome shown in Figure 2 and the chromosome shown in Figure 2(b). Figure 2(a) shows the delivery schedule.

3.3.2. Initial Population Generation. Choosing an appropriate population size helps obtain the optimal calculation speed and most accurate solution. Therefore, this paper uses random rules to generate a set of initial populations with a size of 50 .

3.3.3. Fitness Function. The fitness function is a standard for measuring individuals in the population. In this model, those individuals that satisfy the constraints and the objective function are retained, and others are eliminated. The goal of this paper is to maximize the profit of community group purchase enterprises. To help solve this problem, this paper uses the negative value of the objective function as the fitness function. Therefore, the fitness function of this paper is as follows:

$$
f_{1}^{\prime}=- \text { maxobjective function. }
$$

3.3.4. Crossover. Crossover plays an important role in GA. The principle of the crossover operator is to simulate the process of chromosome repair and gene recombination in biological genetic evolution. It enables the algorithm to obtain a larger gene space, as is shown in Figure 2. We randomly select the gene values of 3 genes in parent 1 and copy them to the same gene of the offspring. Then, the genes in parent 2 with the same gene value as the selected gene from parent 1 are removed. Finally, the formation offspring are obtained by combining parent 1 and parent 2 (see Figure 3).

3.3.5. Mutation. To obtain a global solution, we destroy part of the route so that the individuals in the new group have diversity. We randomly select genes on two loci from the parent and then swap the genes on these two loci to obtain the offspring (see Figure 4).

\section{Results and Discussion}

In this section, this paper assumes that a strawberry planting enterprise in Kunming sells on a community group purchase website. At the same time, the community group purchase enterprise provides delivery services for communities. To investigate the relationship between these objectives, we carry out numerical experiments on randomly generated instances to evaluate the performance of the established model. All experiments are conducted on a computer with an Intel Core i5 $2.5 \mathrm{GHz}$ processor and $8 \mathrm{~GB}$ of memory.

In the first part, we first detail the parameter settings and base instance generation by taking reality into account. Then, accurate algorithm by LINGO and heuristic algorithm based on GA by MATLAB are used to solve these generated instances and compare running times in the next part. In part three, we compare two modes of transportation: refrigerated vehicles and nonrefrigerated vehicles. Parts four and five are devoted to examining the impact of packaging parameters on the overarching goal and exploring how to further reduce distribution costs.

\subsection{Parameter Settings and Base Instance Generation}

4.1.1. Vehicle-Related Parameter Setting. In the practical application at hand, the vehicle type is often different for refrigerated vehicles and nonrefrigerated vehicles. This leads to different load and dead-weight values for the vehicle and different coefficients of calculating fuel costs. The relevant parameter settings are shown in Tables 1 and 2. The same parameters independent of vehicle type are set as follows: $T_{s}=22, V_{0}=600, f=0.7$, and $p=60$.

4.1.2. Package-Related Parameter Setting. At present, the thermal insulation packages used in the market are mostly divided into four different types: cartons, foam cartons, combined carton-and-foam packaging, and composite cartons. Generally, cartons are the cheapest and have the lowest insulation effect, and the combined carton-and-foam packaging type is expensive but has the best insulation effect. The packaging types $b$ are set to $\{1,2,3,4\}$. The value of the unit packaging cost $g_{b}$, the packaging thermal resistance $R_{b}$, and the temperature inside the packaging $T_{b}^{0}$ correspond to the packaging type $b$. Table 3 presents the detailed parameter generation for the packaging.

4.1.3. Base Instance Generation. A base instance is generated based on an investigation of a real enterprise setup. It includes a distribution center, 6 distribution points, and 3 different types of nonrefrigerated vehicles to meet different distribution requirements. We can obtain a great deal of information from the Internet. This information includes the location of the community, which can calculate the distance $d_{i j}$ from node $i$ to node $j$. The vehicle speed $v_{i j}$ is randomly generated from $U[30,80]$. 


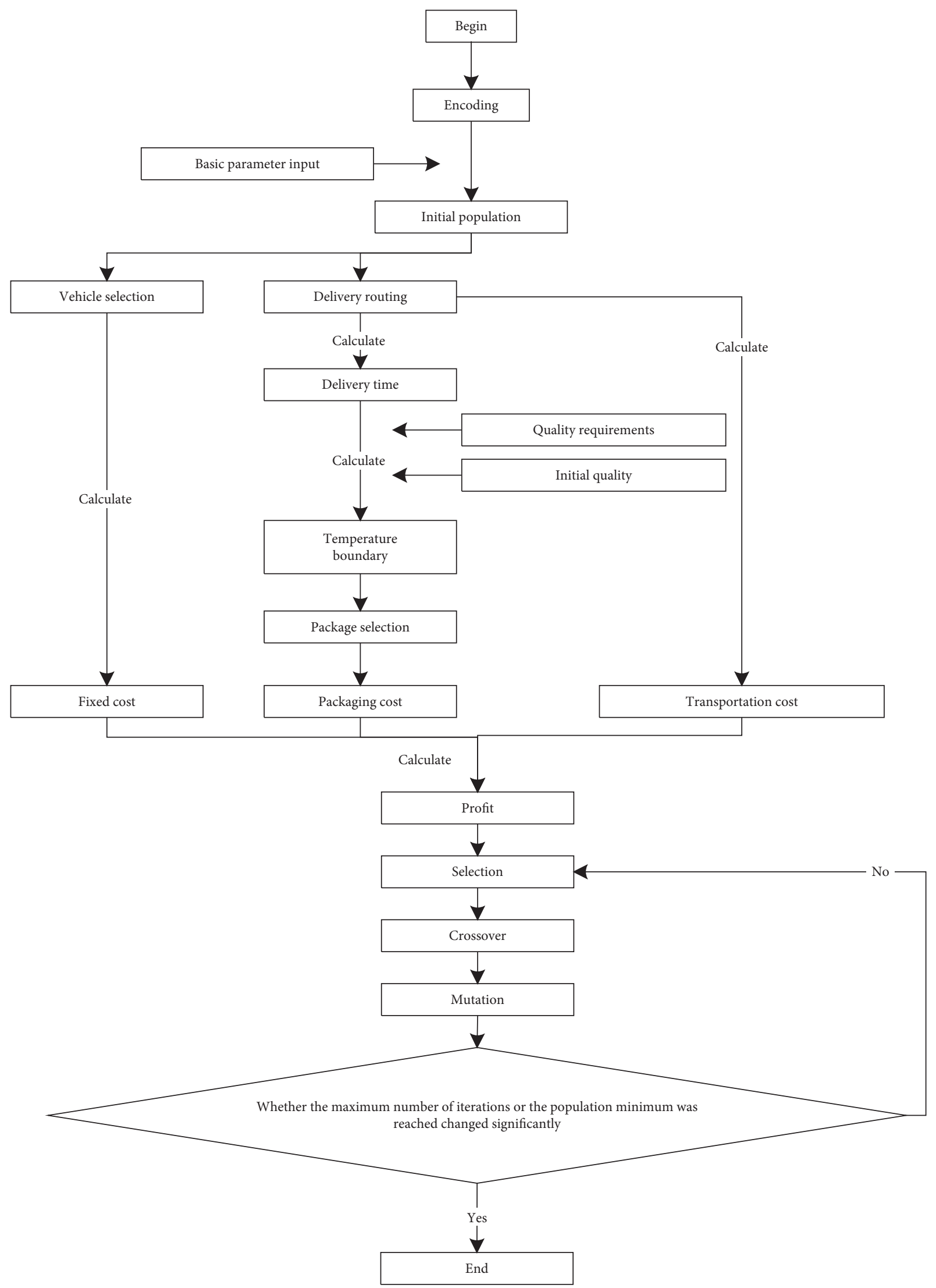

FIgURE 1: Flowchart of the heuristic algorithm based on the genetic algorithm. 


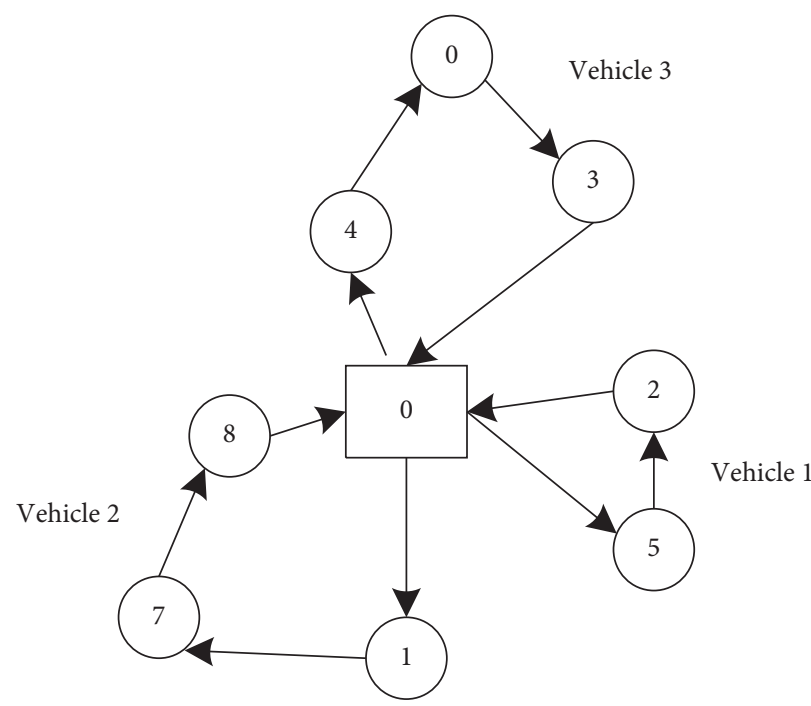

(a)

\begin{tabular}{|c|c|c|c|c|c|c|c|}
\hline 5 & 2 & 1 & 7 & 8 & 0 & 4 & 3 \\
\hline
\end{tabular}

(b)

Figure 2: Delivery schedule and chromosome structure. (a) Delivery schedule. (b) Chromosome structure.

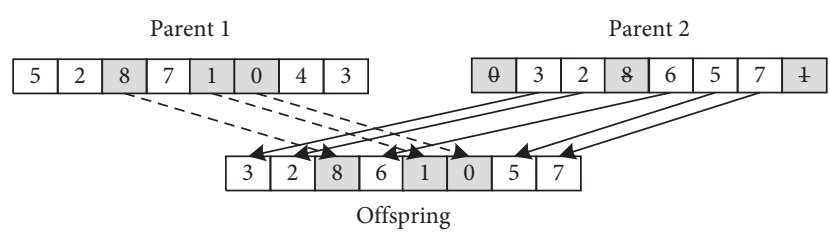

Figure 3: Map of crossover operator.

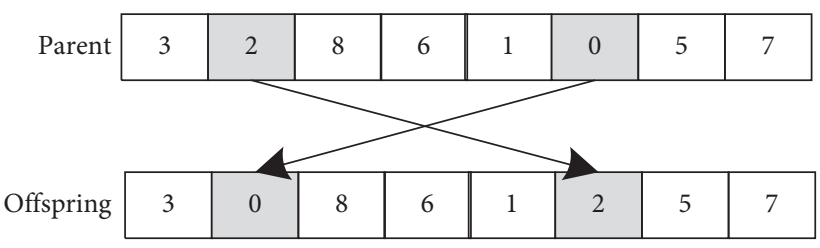

FIgURE 4: Map of mutation operator.

TABLE 1: Parameter setting for nonrefrigerated vehicles.

\begin{tabular}{lccc}
\hline Parameter & \multicolumn{3}{c}{ Value } \\
\hline$k$ & 1 & 2 & 3 \\
$f$ & 218 & 258 & 458 \\
$G$ & 1690 & 1615 & 4185 \\
$a_{0}$ & 1.177 & 1.177 & 1.177 \\
$b_{0}$ & 0.069 & 0.069 & 0.110 \\
$c_{0}$ & 0.001 & 0.001 & 0.000 \\
$w$ & 2250 & 2535 & 3825 \\
\hline
\end{tabular}

4.2. Contrast Analysis between Accurate Algorithm and Heuristic Algorithm Based on GA. To show the effect of different algorithm on the model operation, we generally use LINGO to grogram the exact algorithm and use MATLAB to grogram the heuristic algorithm based on GA. We increase the distribution points from 6 to 10 and generate five data
TABle 2: Parameter setting for refrigerated vehicles.

\begin{tabular}{lccc}
\hline Parameter & \multicolumn{3}{c}{ Value } \\
\hline$k$ & 1 & 2 & 3 \\
$f$ & 268 & 368 & 468 \\
$G$ & 1485 & 3500 & 4380 \\
$a_{0}$ & 0.012 & 1.369 & 1.749 \\
$b_{0}$ & 0.061 & 0.061 & 0.061 \\
$c_{0}$ & 0.014 & 0.014 & 0.014 \\
$w$ & 2680 & 4580 & 4950 \\
\hline
\end{tabular}

TABLE 3: Parameter settings for different types of packages.

\begin{tabular}{lcccc}
\hline Parameter & \multicolumn{5}{c}{ Value } \\
\hline$b$ & 1 & 2 & 3 & 4 \\
$T_{b}^{0}$ & 6.5 & 4.5 & 4 & 4.5 \\
$R_{b}$ & 0.163 & 0.259 & 4.463 & 0.257 \\
$\beta_{b}$ & 0.278 & 0.278 & 0.278 & 0.278 \\
$h_{b}$ & 0.75 & 0.75 & 0.75 & 0.75 \\
$g_{b}$ & 2.25 & 3.38 & 11.27 & 7.61 \\
$L_{b}$ & 335 & 335 & 335 & 335 \\
\hline
\end{tabular}

sets to compare the running times of the accurate algorithm and heuristic algorithm based on GA. These comparison results from accurate algorithm and heuristic algorithm based on GA appear in Table 4.

Table 4 shows that there is a significant difference in running time between accurate algorithm and heuristic algorithm based on GA in the calculation of these instances. Due to the NP-hard nature of our model, the solution run time for accurate algorithm by LINGO has an exponential pattern; therefore, accurate algorithm becomes unable to solve the model when there are more than 9 distribution points. In contrast, the running time in MATLAB is less than 2 seconds in the above instance because the problem can be combined with heuristic algorithm based on GA, which provides 
TABle 4: Parameter settings of different types of packages.

\begin{tabular}{lcc}
\hline Data & Accurate algorithm & Heuristic algorithm based on GA \\
Number of distribution points & Time $(\mathrm{s})$ & Time $(\mathrm{s})$ \\
\hline 6 & 823 & 0.126 \\
7 & 1603 & 0.132 \\
8 & 10825 & 1.286 \\
9 & $86400+$ & 0.525 \\
10 & $86400+$ & 1.043 \\
\hline
\end{tabular}

convenience in the use of intelligent optimization algorithms. This indicates that it is possible to solve the problem with a large number of distribution points with the heuristic algorithm based on GA by MATLAB software; the results for the case with 10 distribution points are shown in Table 5.

Table 5 aggregates the results of the total profit and delivery route and the choice of packaging type. It can be seen that the final total profit is 38343 . Vehicle 2 is chosen to provide delivery services for 10 community demand nodes. The route of the vehicle is 2-9-10-7-4-5-6-1-3-8, and the products delivered to all demand points are packed in cartons.

4.3. Contrast Analysis between Refrigerated and Nonrefrigerated Vehicles. To determine under what circumstances nonrefrigerated thermal insulation packaging is superior to refrigerated vehicles in terms of transporting fresh agricultural products, we set up two cases for the two types of vehicles. For refrigerated vehicles, we can obtain the calculation model for transporting fresh products in refrigerated vehicles from Shui and Li. In practical applications involving nonrefrigerated vehicles, we implicitly assume that the unit packaging cost and refrigerant costs are dependent on the purchase quantity and the manufacturer. We vary the cost of unit packaging and refrigerant in the case of nonrefrigerated packaging. We set up the following cases: Case 1 is identical to the base case with the only difference being that the unit packaging cost increases by $5 \%$, and Case 2 is identical to the base case with the only difference that the unit packaging cost increases by $10 \%$. Case 3 is identical to the base case with the only difference that the unit refrigerant cost increases by $5 \%$. Case 4 is identical to the base case with the only difference that the unit refrigerant cost increases by $10 \%$. The calculated results are shown in Table 6, and the contrast is shown in Figure 5.

We can observe from Figure 5 that the total profits are higher in Case 4, Case 1, and the base case than in Case 5. Nonrefrigerated thermal insulation packaging is superior to refrigerated vehicles under normal circumstances. Simultaneously, the opposite occurs when the unit packaging cost or refrigerant cost is relatively high in small-scale instances. To further investigate the relationship between these costs, we present Figure 6, which shows the percentage of the total cost of using an unrefrigerated vehicle according to Table 6. It clearly reflects which costs are predominant in the total cost.

We notice in Figure 6 that in Cases 1 and 3 and the base case, the cost of using nonrefrigerated vehicles is lower than that of using refrigerated vehicles. Neither the cost of the phase-change refrigerant nor the cost of packaging accounts for more than $50 \%$ of the total cost in these cases. A trade-off exists between the packaging cost and the refrigerant cost in the total cost. This consideration could make nonrefrigerated thermal insulation packaging superior to refrigerated vehicles in terms of transporting fresh agricultural products.

4.4. Trade-Off Cost between Packaging, Refrigerant, and Transportation. In this section, we analyze the impact of the trade-off between total profit and packaging parameters in the last-mile delivery problem. Two common situations arise when we consider the choice of packaging mode in reality. In the first situation, the distribution center selects the least expensive packaging to reduce the packaging cost. In the second situation, the distribution center chooses the best but more expensive packaging. To test the impact of these two situations on the total profit when nonrefrigerated vehicles with thermal insulation packaging are used to transport fresh produce, we set up seven scenarios involving a series of gradual conversions from the first situation to the second situation. By changing the thickness of the packaging, which increases $5 \mathrm{~mm}$ to $35 \mathrm{~mm}$ at intervals of $5 \mathrm{~mm}$, we further change the packaging thermal resistance. Based on realistic factors, packaging with the same specifications has different unit packaging costs under different thicknesses. The relevant parameter settings are shown in Table 7.

From Figures 7 and 8, we have the following observations: (1) The cost of the phase-change refrigerant decreases gradually with increasing packaging thickness. (2) The packaging cost decreases at first and then continuously increases when the packaging thickness increases. (3) Transportation costs vary with the thickness of the packaging, and an overall downward trend is present. (4) Total profit increases at first and then starts to obviously decrease, and the profit reaches the optimal level when each packaging thickness is $10 \mathrm{~mm}$. The above observations can be explained by the following: (1) There is an inverse relationship between the thickness of the packaging and the refrigerant quality, which is lower with thicker packaging. (2) The choice of providing multiple insulation methods with the same thickness is beneficial to businesses in terms of increased profits. (3) The choice among multiple packaging modes affects the choice of vehicle routing for distribution. Because the insulation effect increases with increasing thickness, there are more options when we consider the insulation effect in planning vehicle routing under this change. The 
TABLE 5: The heuristic algorithm based on GA by MATLAB calculation results.

\begin{tabular}{|c|c|c|c|c|c|c|c|c|c|c|}
\hline Time: & \multicolumn{10}{|c|}{0.137 seconds } \\
\hline Total profit & \multicolumn{10}{|c|}{38343} \\
\hline Candidate & \multirow{2}{*}{\multicolumn{3}{|c|}{1}} & \multicolumn{4}{|c|}{2} & \multicolumn{3}{|c|}{3} \\
\hline Delivery route & & & & & $2-9-10$ & 5-6-1-3- & & & & \\
\hline$z_{i}$ & 1 & 2 & 3 & 4 & 5 & 6 & 7 & 8 & 9 & 10 \\
\hline$v^{i}$ & 1 & 1 & 1 & 1 & 1 & 1 & 1 & 1 & 1 & 1 \\
\hline$Q_{i}^{b}$ & 15.58 & 16.24 & 5.22 & 1.46 & 6.52 & 6.023 & 4.32 & 15.48 & 5.48 & 4.82 \\
\hline
\end{tabular}

TABLE 6: Calculation results for each case.

\begin{tabular}{|c|c|c|c|c|c|c|}
\hline & \multicolumn{5}{|c|}{ Instance of nonrefrigerated vehicle } & \multirow{2}{*}{ Instance of refrigerated vehicle } \\
\hline & Base case & Case 1 & Case 2 & Case 3 & Case 4 & \\
\hline \multirow[t]{3}{*}{ Profit } & 25373.6 & 52542.1 & 24916.6 & 25217.7 & 25041.8 & 25172.11 \\
\hline & & centage of th & tal cost of us & an unrefrige & d vehicle & \\
\hline & Base case $(\%)$ & Case $1(\%)$ & Case $2(\%)$ & Case $3(\%)$ & & Case $4(\%)$ \\
\hline Fixed cost & 16.47 & 14.95 & 12.23 & 14.61 & & 13.15 \\
\hline Packaging cost & 21.00 & 37.91 & 51.24 & 18.68 & & 16.81 \\
\hline Refrigerant cost & 37.53 & 26.24 & 18.92 & 44.47 & & 50.02 \\
\hline Fuel cost & 25 & 20.91 & 17.84 & 22.25 & & 20.02 \\
\hline
\end{tabular}

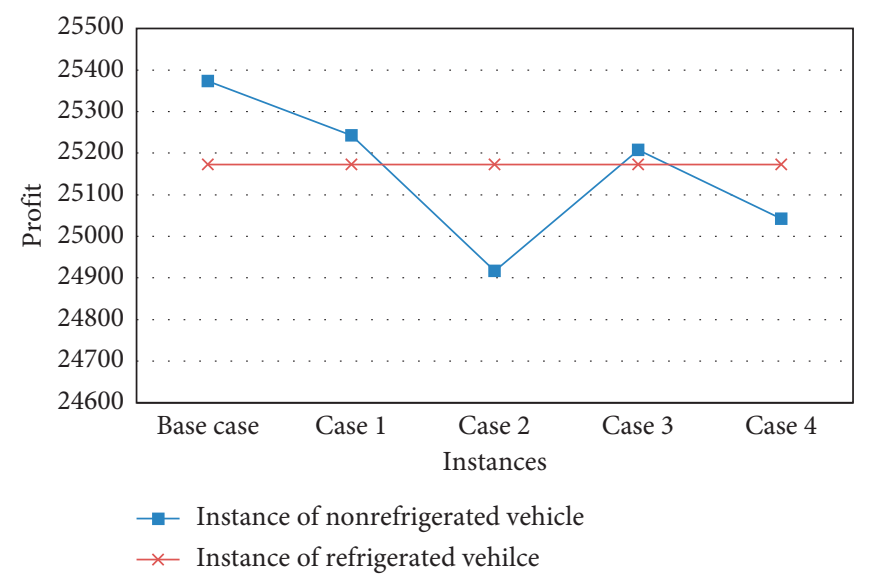

FIgURE 5: Comparison of nonrefrigerated and refrigerated vehicle cases.

distribution route with a lower distribution cost is selected to reduce the transportation cost after we consider the trade-off between the insulation effect and the distribution sequence. (4) We find that there is a trade-off between the objective value and the packaging thickness. On the one hand, this may be because packaging that is too thin is not conducive to maintaining the required distribution temperature, resulting in the need for more phase-change refrigerants to maintain it. This may result in an increase in the total cost of the insulation packaging. On the other hand, higher-priced, thicker packaging more often has a better thermal insulation effect, leading to a reduction in the required refrigerant quality. However, this may lead to an increase in the total packaging cost if the utility of increasing the thickness of packaging exceeds the utility of reducing the phase-change refrigerants. Therefore, the packaging for fresh food distribution should not be too thick or too thin; this choice is determined jointly by the cost of phase-change refrigerants and packaging and the latent heat of phase change of the refrigerants. In summary, the overall result shows that packaging thickness significantly impacts total profit, and the option to choose from multiple packaging modes is beneficial in terms of optimizing the distribution cost with nonrefrigerated vehicles.

4.5. Selection of Insulation Packaging Parameters. To further explore the impact of parameters on the multiple packaging choices, we generate a new class of cases including 3 scenarios. The first scenario is identical to the base case with the only difference being that the thickness of the selected packaging is set to $15 \mathrm{~mm}$ from $10 \mathrm{~mm}$. The second scenario 


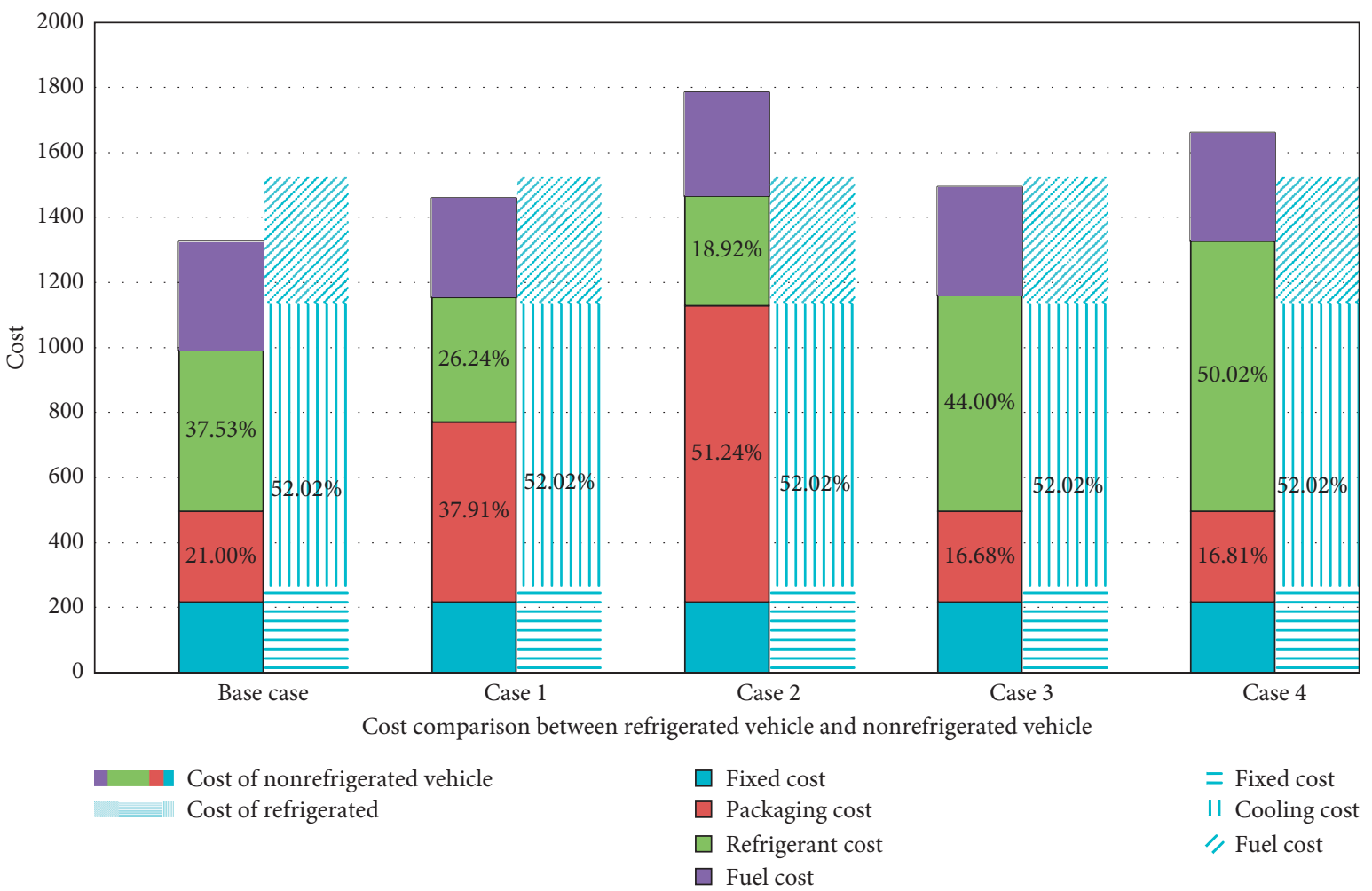

FIgURE 6: Cost comparison of refrigerated and nonrefrigerated vehicles.

TABLe 7: Parameter setting for unit packaging cost and packaging thermal resistance.

\begin{tabular}{|c|c|c|c|c|c|}
\hline Scenarios & $b$ & 1 & 2 & 3 & 4 \\
\hline \multirow{2}{*}{1} & $g_{b}$ & 1.18 & 1.27 & 9.15 & 5.49 \\
\hline & $R_{b}$ & 0.082 & 0.130 & 4.020 & 0.131 \\
\hline \multirow{2}{*}{2} & $g_{b}$ & 2.25 & 3.38 & 11.27 & 7.61 \\
\hline & $R_{b}$ & 0.163 & 0.259 & 4.463 & 0.257 \\
\hline \multirow{2}{*}{3} & $g_{b}$ & 4.37 & 5.49 & 13.38 & 9.72 \\
\hline & $R_{b}$ & 0.246 & 0.390 & 4.901 & 0.412 \\
\hline \multirow{2}{*}{4} & $g_{b}$ & 6.48 & 7.16 & 15.49 & 11.83 \\
\hline & $R_{b}$ & 0.328 & 0.520 & 5.348 & 0.534 \\
\hline \multirow{2}{*}{5} & $g_{b}$ & 8.59 & 9.72 & 17.61 & 13.94 \\
\hline & $R_{b}$ & 0.409 & 0.649 & 5.790 & 0.655 \\
\hline \multirow{2}{*}{6} & $g_{b}$ & 10.7 & 11.83 & 19.72 & 16.06 \\
\hline & $R_{b}$ & 0.492 & 0.779 & 6.233 & 0.777 \\
\hline \multirow{2}{*}{7} & $g_{b}$ & 18.17 & 13.94 & 21.83 & 18.17 \\
\hline & $R_{b}$ & 0.574 & 0.901 & 6.676 & 0.923 \\
\hline
\end{tabular}

is identical to the base case with the only difference that service quality requirements are set to 45 to 75 . The third scenario is identical to the base case with only the difference that the latent heat of phase change is set to 335 to 200 . The results of the calculation are shown in Table 8 .

We can observe from Table 8 that packaging type 3 is not selected in any of the scenarios, which means that the distribution center usually uses cheaper packaging instead of using better, higher-priced packaging to save on distribution costs. Essentially, in choosing packaging, the community group purchase enterprise faces a trade-off between shelf life and cost, and as long as the less expensive packaging with the appropriate quality of phase-change refrigerant can ensure delivery quality requirements, the enterprise does not choose more expensive packaging that can extend shelf life of perishable products.

However, the distribution center chooses better, higherpriced packaging to prevent the food products from decaying when we have higher quality requirements or when the latent heat of the phase-change refrigerant is lower in scenario 1 and scenario 2. This conclusion has also been drawn by Li [15]. We can also see that the packing mode changes in scenario 1 . The results indicate that the multiple packaging and thickness options have a significant impact 


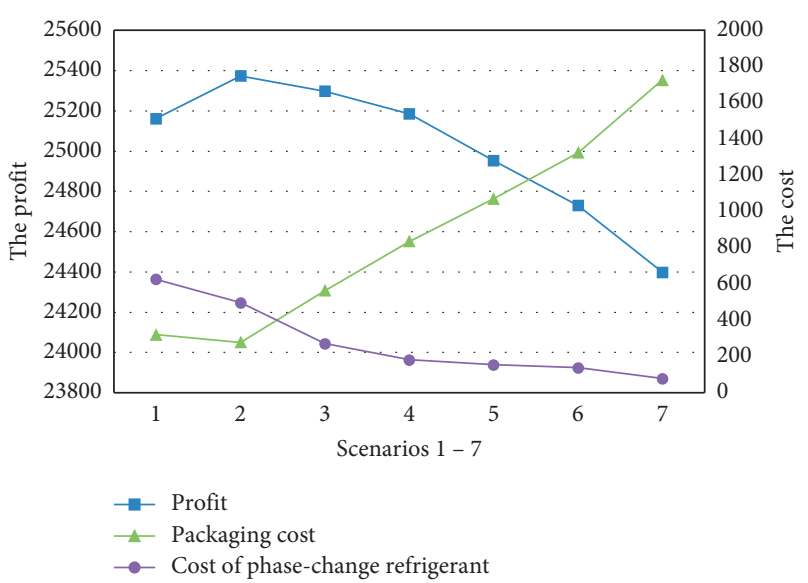

Figure 7: Cost and profit trends.

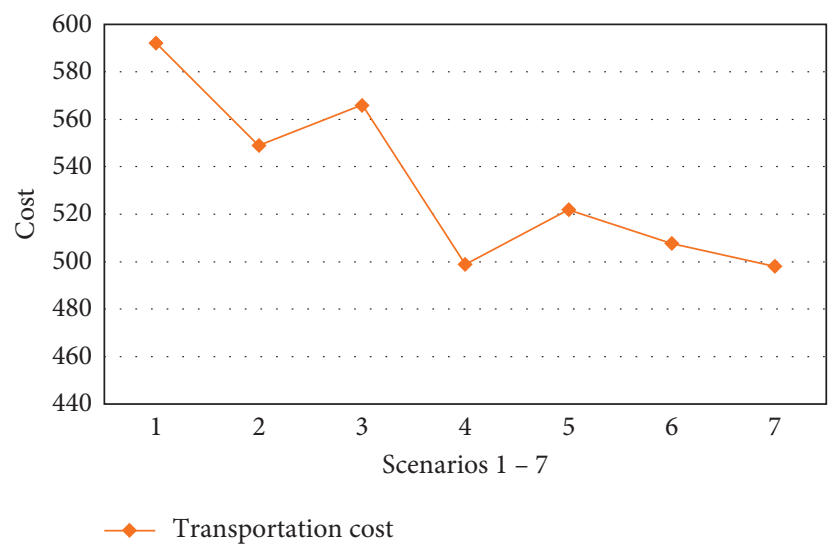

FIgURE 8: Transportation cost trend.

TABLE 8: Results of the calculation for each scenario.

\begin{tabular}{|c|c|c|c|c|c|c|c|}
\hline & \multirow{3}{*}{ Profit } & \multicolumn{6}{|c|}{ Community distribution points } \\
\hline & & 1 & 2 & 3 & 4 & 5 & 6 \\
\hline & & \multicolumn{6}{|c|}{ Selection of packing mode } \\
\hline Base case & 25373.61 & 1 & 1 & 1 & 1 & 1 & 1 \\
\hline Scenario 1 & 25480.18 & 1 & 1 & 2 & 2 & 1 & 1 \\
\hline Scenario 2 & 25384.71 & 2 & 2 & 2 & 2 & 2 & 4 \\
\hline Scenario 3 & 25252.47 & 1 & 2 & 2 & 2 & 2 & 4 \\
\hline
\end{tabular}

on the final result and can further improve distribution costs and profits.

\section{Conclusion}

This paper proposes a collaborative optimization mechanism for different insulation package selections and cold chain logistics distribution paths under the community group purchase mode. For small-scale numerical examples, LINGO software is used to solve the problem. On this basis, a heuristic algorithm based on a genetic algorithm is proposed for large-scale numerical examples, and MATLAB is used to solve and prove its effectiveness.
Through parameter analysis, the following conclusions are drawn. First, the large-scale cases are more suitable for solution with the MATLAB software than with LINGO. Second, the use of nonrefrigerated thermal insulation packaging is superior to the use of refrigerated vehicles in terms of transporting fresh agricultural products when neither the cost of the phase-change refrigerant nor the cost of packaging accounts for more than $50 \%$ of the total cost. Third, the overall result shows that the option to use multiple packaging thicknesses is beneficial to improve the distribution cost of nonrefrigerated vehicles. Furthermore, choosing better packaging is beneficial in terms of reducing transportation costs. Finally, distribution centers usually use cheap packaging with high-quality refrigerants to ensure the quality of fresh produce and a low total cost.

In regard to future research, we will further consider multiproduct fresh food distribution in this way. In addition, the choice of refrigerant type should be taken into account. To further reduce the distribution cost, in the case of fewer fresh orders, the joint distribution of ordinary packages and fresh agricultural product packages by nonrefrigerated vehicles can be considered in the context of the community group purchase model.

\section{Data Availability}

The data used to support the findings of this study are available from the corresponding author upon request.

\section{Conflicts of Interest}

The authors declare that there are no conflicts of interest regarding the publication of this paper.

\section{Acknowledgments}

The authors acknowledge the National Natural Science Foundation of China (Grant no. 71462024).

\section{References}

[1] Y.-M. Li, J.-H. Jhang-Li, T.-K. Hwang, and P.-W. Chen, "Analysis of pricing strategies for community-based group buying: the impact of competition and waiting cost," Information Systems Frontiers, vol. 14, no. 3, pp. 633-645, 2012.

[2] Q. Li, X. Li, X. Xiu et al., "Study on consumers' community group purchase based on the integration of SOR and commitment-trust theory," Journal of Xi'an Jiaotong University, vol. 40, no. 2, pp. 25-35, 2020.

[3] Y. Liu, M. Jiang, L. Li et al., "Research on evaluation and selection of group purchase distribution mode in fresh Community1," International Journal of Business and Social Science, vol. 10, no. 9, pp. 101-109, 2019.

[4] Y. Wang and W. Qiu, "Optimization of distribution path for community group buying considering customer satisfaction," IOP Conference Series: Materials Science and Engineering, vol. 10, no. 1, Article ID 012008, 2020.

[5] H. G. Lee and S. Yoo, "Changes in the physical properties of frozen cooked rice depending on thermal insulation levels of packaging during freeze-thaw," Journal of Food Science, vol. 85, no. 12, pp. 4342-4350, 2020. 
[6] W. Fang, H. Song, D. Wu et al., "Temperature field simulation of multi-temperature zone thermal insulation box based on fluent," Packaging Engingeering, vol. 41, no. 3, pp. 62-68, 2020.

[7] Q. Li, P. Yu, X. Wu et al., "Shelf life extending packaging, inventory control and grocery retailing," Production and Operations Management, vol. 26, no. 7, pp. 1369-1382, 2017.

[8] E. Oró, L. Miró, and L. F. Cabeza, "Thermal analysis of a low temperature storage unit using phase change materials without refrigeration system," International Journal of Refrigeration, vol. 35, no. 6, pp. 1709-1714, 2012.

[9] S. P. Singh, G. Burgess, J. Singh et al., "Performance comparison of thermal insulated packaging boxes, bags and refrigerants for single-parcel shipments," Packaging Technology and Science, vol. 21, no. 1, pp. 25-35, 2008.

[10] P. Amorim and B. Almada-Lobo, "The impact of food perishability issues in the vehicle routing problem," Computers \& Industrial Engineering, vol. 67, pp. 223-233, 2014.

[11] C.-I. Hsu, S.-F. Hung, H.-C. Li et al., "Vehicle routing problem with time-windows for perishable food delivery," Journal of Food Engineering, vol. 80, no. 2, pp. 465-475, 2007.

[12] M. Rabbani, A. Farshbaf-Geranmayeh, N. Haghjoo et al., "Vehicle routing problem with considering multi-middle depots for perishable food delivery," Uncertain Supply Chain Management, vol. 4, no. 3, pp. 171-182, 2016.

[13] D. S. Byung and D. A. Young, "A vehicle routing problem of both refrigerated- and general-type vehicles for perishable food products delivery," Journal of Food Engineering, vol. 169, pp. 61-71, 2016.

[14] E. Dieckmann, B. Nagy, K. Yiakoumetti et al., “Thermal insulation packaging for cold-chain deliveries made from feathers," Food Packaging and Shelf Life, vol. 21, Article ID 100360, 2019.

[15] Y. Li, C. Feng, C. Jean-Francois et al., "The multi-plant perishable food production routing with packaging consideration," International Journal of Production Economics, vol. 221, p. 2020, Article ID 107472.

[16] W. Shui and M. Li, "Integrated pricing and distribution planning for community group purchase of fresh agricultural products," Scientific Programming, vol. 2020, Article ID 8839398, 15 pages, 2020.

[17] B. D. Coleman and J. Victor, "Thermodynamics and departures from Fourier's law of heat conduction," Archive for Rational Mechanics and Analysis, vol. 13, no. 1, pp. 245-261, 1963.

[18] M. Pinto and J. C Pinto, "Optimum reference temperature for reparameterization of the Arrhenius equation. Part 1: problems involving one kinetic constant," Chemical Engineering Science, vol. 62, no. 10, pp. 2750-2764, 2007. 